\title{
Bariatric Surgical Treatment for Obese Adolescents and Children
}

\author{
CHOUDHURY KM ${ }^{\mathrm{a}}$, ZAFAR SMA $^{\mathrm{b}}$
}

\begin{abstract}
Like in adults, the prevalence of childhood and adolescent obesity is increasing globally. The consequences of severe obesity are serious and for that reason the World Health Organization has recognized obesity as a disease. Among the treatment options for obesity comprising dietary restriction, increased physical activities and weight-reduction surgery, only the last one provides a sustained and consistent weightloss in obese patients. Numerous recent studies have validated this fact in favor of bariatric surgeries. Although in spite of having been associated with considerable significant
\end{abstract}

\section{Introduction:}

Obesity is a serious ailment-chronic, progressive and is often life-threatening. In addition to adults, increasing number of children are also afflicted with the disease. Although prevention is the ultimate goal, a substantial number of children and adolescents will have significant comorbidities related to obesity and will require treatment. At present, surgical treatment options are deemed more effective at achieving sustained weight loss and for amelioration of the comorbidities in comparison to nonsurgical weight-loss strategies particularly in severely obese patients. There is mounting body of evidence that supports the use of modern surgical weight loss procedures for carefully selected, extremely obese adolescents. ${ }^{1,2}$ However, one needs to remember that choosing a weight-loss surgical strategy for children and adolescents should be patient-specific based on their age, severity of comorbidities and body mass index (BMI). It should also be borne in mind that the nutritional consequences of the most common bariatric surgical

a. Dr. Kamal M. Choudhury, MS (Pediatric Surgery). Associate Professor and Head, Department of Pediatric Surgery. BIRDEM General Hospital,

b. Prof. Dr. Sheikh Mohammad Abu Zafar, FRCS. Professor, Department of Surgery. BIRDEM General Hospital.

Address of Corresponence: Dr. Kamal M Choudhury, Associate Professor and Head, Department of Pediatric Surgery, BIRDEM General Hospital, E-mail address: kamalemcee@gmail.com complications, these are gaining popularity particularly in morbid obesity. The justification of bariatric surgeries in children and adolescents is still a contentious issue especially in regards to their physiologic growth and development. In many advanced countries, pediatric bariatric surgeries are being done under strict guidelines including India; but in our country, it is still in conceptual stage. Here we present essentially a conceptual framework and clinical guideline for bariatric surgery in adolescents and children in this review.

(Birdem Med J 2015; 5(Supplement 1): 34-42)

procedures are well defined and must be effectively managed with vitamin and mineral supplementation. Among adolescents, however, it is not fully known whether the outcome will be the same as in adults or whether there will be unacceptably high degree of recidivism and long-term nutritional sequelae related to poor compliance or reduced micronutrients absorption. Nonetheless, as severely obese patients are refractory to even the most intensive conventional non-surgical approaches to weight-loss, bariatric surgery is becoming a popular means to treat such morbid adolescents. On the basis of the available evidence, this review proffers a conceptual framework and clinical guidelines for bariatric surgery in adolescent and children.

Common-sense approaches to the treatment of obesity were aimed at limiting food intake, increasing physical activity and modification of behavior. These measures could work for the slightly overweight to minimally obese individuals who are motivated and disciplined to undergo significant life-style change. For severe obese patients, diet and behavior modification and exercise have limited success and a high recidivism rate. ${ }^{3}$ Surgical procedures for the treatment of obesity have been developed during the later decades of twentieth century. Initial bariatric procedures, such as the jejuno-ileal bypass, were designed with limited understanding to the underlying physiologic mechanisms controlling hunger, satiety, caloric absorption, and the bystander effects on the liver and the body as a whole. ${ }^{4}$ However, 
recent weight-loss surgery has become more refined with combinations of intestinal bypass to interfere with caloric absorption and a variety of restrictive procedures and devices to impede food ingestion. Scientists have discovered that an increasing complex array of signals control energy homeostasis but much research work remains to be done to unravel the riddle of obesity.

\section{Definition:}

Obesity is defined as a long-term positive imbalance between energy intake and expenditure, with increased adipose tissue lipid storage and number of fat cells. ${ }^{5}$ Children with BMI at greater than $85^{\text {th }}$ percentile $(>85 \mathrm{P})$ are considered overweight and those with greater than $95^{\text {th }}$ percentile $(>95 \mathrm{P})$ are considered obese. ${ }^{6}$ Those with BMI greater than $99^{\text {th }}$ percentile $(>99 \mathrm{P})$ are considered severely obese.

\section{Incidence:}

Between 1980 and 2010, the incidence of obesity in children has increased to about three-fold in the United States. ${ }^{6}$ In children aged 2 to 5 years, the incidence increased from $5 \%$ to $10.4 \%$ whereas in children aged 6 to 11 years, it increased from $6.5 \%$ to $19.6 \%$, and in adolescents aged 12 to 19 years from $5 \%$ to $18 \% .{ }^{6,7} \mathrm{~A}$ study in 2007 showed that $32 \%$ of children and adolescents (2-19 years old) were overweight $(>85 \mathrm{P}), 17 \%$ were obese $(>95 \mathrm{P})$ and $4 \%$ were severely obese $(>99 \mathrm{P}) .{ }^{8}$

An ICDDR.B survey in 2013 conducted on children between five and 18 years and their mothers showed $14 \%$ children were overweight and $4 \%$ were obese. The study also found that the prevalence of obesity and overweight has increased in children and adolescents from $8.1 \%$ to 12.9 in 2013 for boys and from $8.4 \%$ to $13.4 \%$ in girls. ${ }^{9}$

Another study in 2014 observed that among school aged (6 to 15 years) children in Bangladesh, 3.5\% were obese and $9.5 \%$ were overweight. ${ }^{10}$

\section{Risk factors for obesity in children:}

The risk of obesity accumulates with age. Recent insights into the fetal, neonatal and developmental origins of obesity have implications for clinical evaluation of the adolescent candidate for bariatric surgery. ${ }^{11}$ Epidemiologic studies have established significant link between birth weight and later body mass index in childhood and adulthood. ${ }^{12}$ Lower birth weight increases the risk for obesity and insulin resistance. ${ }^{13}$

Childhood obesity risks are higher in infants of diabetic mothers (with high birth weight) and in infants with early rapid weight gain. Absence or short-duration of breast- feeding confers enhanced risk of obesity in adolescence. Maternal pre-pregnancy overweight and maternal smokings in pregnancy also increase the likelihood of childhood obesity. Obesity among the parents and siblings is an additional risk factor. The odds ratio for persistence of childhood obesity into adulthood is about 3 if one or 10 if both parents are obese. ${ }^{14}$

\section{Comorbidities:}

Potential health consequences of pediatric obesity include insulin resistance (metabolic syndrome) and type-2 diabetes; hypertension, dyslipidemia, hypercholesterolemia, hypercoagulability and endothelial dysfunction; fatty liver disease, gall-stones and nonalcoholic steatohepatitis; sleep apnea, exercise intolerance and asthma; pseudotumor cerebri and polycystic ovary syndrome etc.

Though type-2 diabetes is typically a disease of middleaged obese persons, a significant number (nearly half) of new patients with type-2 diabetes are children. ${ }^{15}$ The course and complications of type- 2 diabetes in children are similar to that seen in adults. Gastric bypass surgery in adolescent has been shown to resolve type-2 diabetes following significant weight loss. ${ }^{16}$ It is now widely agreed that established type- 2 diabetes is a strong indication for bariatric surgery in adolescents. ${ }^{17}$

Pediatric obesity is likely to persist and approximately $70 \%$ to $80 \%$ children may become obese adults (juvenileonset obesity). This cohort of obese patients are particularly vulnerable to develop cardiovascular complications. ${ }^{8}, 18$ At present, cardiovascular risk factors are less strong indications for bariatric intervention in adolescents.

Non-alcoholic steatohepatitis (NASH) is a serious consequence of childhood obesity and is regarded as a cause of chronic liver disease in children. Approximately $9 \%$ of obese children and adolescents have NASH compared with $1 \%$ of the lean population. It may lead to hepatic failure and liver transplantation. ${ }^{19}$ Currently, weight loss is the only treatment for severe steatosis; $\mathrm{NASH}$ is, therefore, considered a strong indication for bariatric surgery. ${ }^{17}$

Sleep apnea, exercise intolerance and asthma are common consequences of pediatric obesity. Obstructive sleep apnea can significantly impair learning abilities with a negative effect on school and work performance. Obstructive sleep apnea improves or resolves completely in adolescent undergoing bariatric surgery. ${ }^{20}$

The most prevalent and debilitating consequences of adolescent obesity are psychosocial and are as 
damaging as the medical morbidities. Emotional comorbidities include low self-esteem, negative body image, sadness \& depression and high-risk behaviors. Social comorbidities include isolation, stigmatization, discrimination and negative stereotyping. These often result in poor school performance and are associated with lower levels of socioeconomic attainment. A recent study has demonstrated improvement in depressive symptoms and quality of life over the first postoperative year following bariatric surgery in adolescents. ${ }^{21}$

\section{Treatment for childhood obesity:}

There are anecdotal opinions that children do not require treatment for obesity because they will grow out of it. However, various data and numerous literatures unequivocally refute such assumption; rather they assert the notion that adult obesity usually begins in childhood and is correlated with the duration and degree of childhood obesity. ${ }^{22}$ Therefore, treatment protocols for obesity should be 'need' related not 'age' related; sick children receive treatment when they have a problem, regardless of their age. ${ }^{22}$

\section{Weight management program:}

Weight reduction for obesity includes three essential core elements: nutrition education, physical activity, and behavioral modification. The program should involve the following personnel in different subspecialties: pediatric endocrinologist, pediatric bariatric surgeon, dietitian, physical trainer, psychiatrist and pediatric nurse.

\section{Surgical procedures for weight loss:}

Bariatric procedures are not unique for children. The procedures used in adults are being adopted in adolescents and children. The consideration that should be emphasized for children and adolescents is that unlike adults they have not completed the growth and maturation. In this regard, few questions needed to be resolved- whether children and adolescents require alternate selection criteria or different postoperative management strategies than adults and whether the longterm nutritional and metabolic outcomes of adolescents will be better or worse than similarly obese adolescents who had undergone bariatric surgery in youth.

The commonly practiced procedures in this cohort are laparoscopic adjustable gastric band (LAGB), sleeve gastrectomy (SG) and Roux-en-Y gastric bypass (RYGB).

History of Pediatric Bariatric Surgery: The earliest report of pediatric bariatric surgery was Randolph's experience with jejuno-ileal bypass. ${ }^{38}$ Though there was dramatic weight loss with improvements in quality of life (QOL), the metabolic complications encountered in this procedure were unacceptable. Greenstein used gastric band as restrictive measure and reported favorable experience with low mortality risk. ${ }^{25}$ A review of the published series included more than 200 adolescents and showed significant excess weight loss during 6-month to 7-year follow-up. ${ }^{17}$ In recent time, bariatric procedures among adolescents are beginning to be studied in a prospective and controlled fashion. One such randomized controlled trial (RCT) among 50 adolescents compared gastric banding (AGB) with lifestyle intervention and showed a weight loss of $34.6 \mathrm{~kg}$ (BMI reduction of $28 \%$ ) in AGB group compared with a 3 -kg loss (BMI reduction of $3 \%$ ) in the life-style group. ${ }^{26}$ However, the high rate of surgical complications that required revision surgery was of considerable concern. A meta-analysis of 8 AGB studies with data on 352 patients (mean BMI $45.8 \mathrm{~kg} / \mathrm{m}^{2}$ ) demonstrated sustained weight loss and complications similar to adult AGB patients. ${ }^{27}$ The systematic review and meta-analysis on 6 RYGB studies including 131 adolescents with mean BMI of $51.8 \mathrm{~kg} / \mathrm{m}^{2}$ reported a significant and sustained decrease in BMI after RYGB procedure. ${ }^{27}$ Serious complications such as anastomotic leak, sepsis, bleeding and thromboembolic events are rarely reported. Another study with 61 adolescents (mean pre-operative BMI of $60.2 \mathrm{~kg} / \mathrm{m}^{2}$ ) who underwent RYGB had a weight loss of $37.4 \%$ one year postoperatively with significant improvements of comorbidities. ${ }^{28}$

The Best Bariatric Surgical Procedure: In children and adolescents, an ideal bariatric procedure is one that would result in durable and substantial weight loss, with minimal risk from short-term procedural complications and long term malnutrition, growth limitation, liver problems or mechanical complications. Significant disagreement exists regarding the best bariatric surgical procedure in children and adolescents. Each of the procedures has advantages achieving significant weight loss with reversal of comorbidities, and disadvantages with unique profile of potential complications and nutritional concerns. Definitive comparative studies often yield equivocal results. ${ }^{29}$ More RCTs and meta-analyses will be required to single out the most optimum procedure. Table-1 depicts a comparison between three common procedures.

Certain issues and factors are crucial in the process of choosing and selecting a bariatric procedure for children and adolescents. Current clinical guidelines for pediatric bariatric procedures are primarily based on the critical analyses of various procedures for both adults and adolescents. ${ }^{30-33}$ In order to frame standardized 
treatment protocol, a brief discussion of the existing bariatric procedures in use in children is presented.

LAGB is an effective procedure for $60 \%$ to $80 \%$ of patients. It is least invasive and most common procedure in Europe and Australia. It is currently being used within the context of an investigational device exemption from the US Food and Drug Administration (FDA) in adolescents younger than 18 years at several US centers. It is effective for patients with a BMI of 50 or less. Excess weight loss is about $50 \%$ over a 2 - to 3 -year period. ${ }^{34-35}$

Table-I

Comparison of bariatric surgical procedures. ${ }^{32-33}$

\begin{tabular}{|c|c|c|c|}
\hline Type of Operation & RYGB & SG & LAGB \\
\hline Anatomy & $\begin{array}{l}\text { Small } 20-30 \mathrm{ml} \text { gastric pouch } \\
\text { Pouch connected to the small } \\
\text { intestine with an outlet }(1-2 \mathrm{~cm}) \\
\text { Gastrointestinal bypass ranging } \\
\text { from } 100-250 \mathrm{~cm} \text { and excluding } \\
\text { food from digestive juices. }\end{array}$ & $\begin{array}{l}\text { Long narrow gastric sleeve }(100 \mathrm{ml}) \\
\text { along the lesser curvature } \\
\text { No intestinal bypass } \\
\text { Majority stomach (fundus and greater } \\
\text { curvature) removed from } 6 \mathrm{~cm} \text { from } \\
\text { pylorus to the angle of His }\end{array}$ & $\begin{array}{l}\text { An adjustable silicone band is } \\
\text { placed around the top part of the } \\
\text { stomach creating a small } 30-60 \mathrm{ml} \\
\text { pouch } \\
\text { A port for adjustment of the band is } \\
\text { placed on the anterior abdominal } \\
\text { wall }\end{array}$ \\
\hline Mechanism & $\begin{array}{l}\text { Food volume is restricted } \\
\text { Mild malabsorption } \\
\text { Negative feedback in the form of } \\
\text { dumping syndrome when sugar or } \\
\text { fats are consumed } \\
\text { Faster release of GLP-1 and PYY } \\
\text { increases early satiety }\end{array}$ & $\begin{array}{l}\text { Food volume is restricted } \\
\text { No malabsorption } \\
\text { No dumping } \\
\text { Good physiologic sense of fullness } \\
\text { from restriction in reduced stomach } \\
\text { Increased GLP-1 and PYY } \\
\text { Decreased ghrelin levels curb appetite }\end{array}$ & $\begin{array}{l}\text { Food volume is restricted } \\
\text { Adjustable tightness of band delay } \\
\text { pouch emptying and prolong sense } \\
\text { of fullness }\end{array}$ \\
\hline Weight loss & $\begin{array}{l}\text { Excess weight loss } 65 \%--70 \% \\
\text { Lost within } 12-18 \text { months } \\
\text { Initially greater weight loss, } \\
\text { which levels off }\end{array}$ & $\begin{array}{l}\text { Excess weight loss } 33 \%--83 \% \\
\text { Lost within } 12-24 \text { months } \\
\text { Initially greater weight loss, which } \\
\text { levels off }\end{array}$ & $\begin{array}{l}\text { Excess weight loss about } 50 \% \\
\text { Lost over } 36 \text { months } \\
\text { Weight loss gradual over first year } \\
\text { but similar to other procedures by } \\
2-3 \text { years }\end{array}$ \\
\hline $\begin{array}{l}\text { Long-term Dietary } \\
\text { Modification }\end{array}$ & $\begin{array}{l}3 \text { small high-protein meals per } \\
\text { day } \\
\text { Must avoid sugar \& fats to } \\
\text { prevent dumping syndrome } \\
\text { Vitamin /protein deficiency } \\
\text { preventable with supplements. }\end{array}$ & $\begin{array}{l}\text { No dumping, no diarrhea } \\
\text { Weight regain may be more likely } \\
\text { than in other procedures if dietary } \\
\text { modifications not adopted for life }\end{array}$ & $\begin{array}{l}\text { Certain dense foods can get stuck if } \\
\text { not chewed well (causing pain and } \\
\text { vomiting) } \\
\text { No liquids with meals }\end{array}$ \\
\hline $\begin{array}{l}\text { Nutritional } \\
\text { supplements }\end{array}$ & $\begin{array}{l}\text { Multivitamin } \\
\text { Vitamin } \mathrm{B}_{12} \\
\text { Calcium } \\
\text { Iron }\end{array}$ & $\begin{array}{l}\text { Multivitamin } \\
\text { Calcium }\end{array}$ & $\begin{array}{l}\text { Multivitamin } \\
\text { Calcium }\end{array}$ \\
\hline Potential Problems & $\begin{array}{l}\text { Dumping syndrome } \\
\text { Stricture } \\
\text { Ulcers } \\
\text { Bowel obstruction } \\
\text { Anemia } \\
\text { Vitamin/mineral deficiencies } \\
\text { Anastomotic leak } \\
\text { Weight regain } \\
\text { Technical challenging }\end{array}$ & $\begin{array}{l}\text { Nausea \& vomiting } \\
\text { Heartburn } \\
\text { Inadequate weight loss } \\
\text { Weight regain } \\
\text { Staple line leak } \\
\text { Additional procedure may be needed } \\
\text { to obtain adequate weight loss } \\
\text { Technically easy }\end{array}$ & $\begin{array}{l}\text { Slow weight loss } \\
\text { Slippage } \\
\text { Erosion } \\
\text { Infection } \\
\text { Port problems/ device malfunction } \\
\text { Additional procedure may be } \\
\text { needed for adequate weight loss } \\
\text { Technically easy }\end{array}$ \\
\hline Hospital Stay & 2-3 days & 1-2 days & Overnight ( $<1$ day) \\
\hline Time out of school & 2-3 weeks & $1-2$ weeks & 1 week \\
\hline Operating time & 2 hours & 1.5 hour & 1 hour \\
\hline Insurance coverage & $\begin{array}{l}\text { Most payers will cover the RYGB } \\
\text { even in adolescents }\end{array}$ & $\begin{array}{l}\text { Third-party payers have been } \\
\text { reluctant to cover the SG, especially } \\
\text { in children }\end{array}$ & $\begin{array}{l}\text { The AGB is not FDA approved for } \\
\text { use in patients aged } 18 \text { years or } \\
\text { younger. }\end{array}$ \\
\hline
\end{tabular}


LAGB has a short learning curve. The complication rate is $6 \%$ to $15 \%$ and most are related to mechanical issues, such as the band's position, pouch enlargement, or port and catheter problems.

In comparison, the RYGB provides significant and rapid weight loss $(65 \%$ to $70 \%)$ in most adolescent patients. However, it carries a slightly higher mortality rate. ${ }^{36-37}$ The excellent weight loss needs to be balanced against the serious short- and long-term surgical complications including dumping syndrome, marginal ulcers, internal hernias, micronutrients and vitamin deficiencies. It is the most common bariatric procedure performed in the USA.

The SG is a technically simple procedure and is the most acceptable first-line option in adult obese patients. Excess weight loss ranging from $33 \%$ to $83 \%$ has been reported. ${ }^{38}$ In this procedure, complications are minimal and manageable. American Society for Metabolic and Bariatric Surgery (ASMBS) has accepted the SG as an approved bariatric surgical procedure for adults. ${ }^{39}$ The importance of SG in adolescents is its successful application as a salvage operation for obese children who do not respond sufficiently to or have mechanical complications with LAGB. In addition, short-term data of SG as an initial procedure in children and adolescents are quite encouraging. ${ }^{40}$ Laparoscopic SG does involve much less anatomic and physiologic derangement than that of RYGB and is an attractive alternative in the adolescents. SG decreases ghrelin level which should be a concern as it may adversely affect growth and development of the adolescent patients.

\section{New devices and new procedures:}

Several new devices and one new procedure are currently under development. Presently, the AGB is the only weight loss device in use. Devices may be an ideal alternative to weight-loss surgical procedure because they are minimally invasive and may have lower morbidity. Also, the devices are adjustable, reversible and removable. Intragastric balloon and Endosleeeve are regarded as temporary device while Vagal-blocking device and gastric stimulator are considered as semipermanent device

Intragastric Balloon: This is a temporary intraluminal device as it is removed or changed on a regular basis. It would be attractive in children because it could help children attain a healthy body fat proportion when its use might be discontinued. ${ }^{41}$ The balloon is designed to be inflated in the stomach and causes a continual sensation of gastric fullness and satiety. The device is adjustable that will allow the balloon size to be titrated to the size of the patient and to the desired effect.

Endosleeve: It consists of a tube made of impermeable material placed along the lumen of duodenum that extend distally for about $60 \mathrm{~cm}$ thus creating a mechanical bypass for ingested food resulting in impediment with absorption. ${ }^{42}$ It also prematurely initiates satietyinducing neuro-hormonal feed-back loop when PYY and GLP-1 are released from the distal ileum causing early satiety. It may be used for 6 months; after which it is removed and replaced endoscopically.

Vagal-Blocking Device: It is known that vagal afferents to the central nervous system (CNS) can control appetite. Blocking the afferent pathways by electronic device and controlling the frequency, amplitude and wave form can result in optimum effects in satiety and consequent weight loss. ${ }^{43}$

Gastric Stimulator: Gastric stimulator induces weight loss by interfering with ghrelin production or by simulating the sensation of a full stomach via vagal afferents. $^{44}$

Gastric Plication: It is a new bariatric surgical procedure and involves plicating the greater curve, fundus and body of the stomach to create a tube along the lesser curvature. ${ }^{45}$ It mimics the SG without excision of the stomach.

\section{Suggested protocol for management of obesity in adolescents and children:}

As non-operative weight loss treatment did not have desired success particularly in patients with severe obesity, it became apparent that better and more effective mode of treatment must be developed. However, many physicians caring morbidly obese children and adolescents, though frustrated with the ineffectiveness of current weight loss program, are reluctant to condone surgical interventions. This is because evidence regarding the outcome of bariatric procedures in adolescents and children is just beginning to be published and lack clear-cut consensus. However, the pediatric committee for the ASMBS published the guidelines $^{44}$ that recommended criteria for patient selection (aged 13 to 19 years) for bariatric surgery:

1. BMI greater than or equal to $40 \mathrm{~kg} / \mathrm{m}^{2}$ with or without comorbidities namely, hypertension, insulin 
resistance, glucose intolerance, dyslipidemia and mild sleep apnea (apnea-hypopnea index or AHI $>5$ ).

2. BMI greater than or equal to $35 \mathrm{~kg} / \mathrm{m}^{2}$ with major comorbidities namely, type- 2 diabetes, moderate to severe sleep apnea $(\mathrm{AHI}>15)$, pseudotumor cerebri, severe nonalcoholic steatohepatitis (NASH).

3. Must have failed 6 months intensive and professionally monitored non-operative weight loss program.

4. Must have psychological maturity to understand implications of surgery and be competent to assent to the procedure and be compliant with postoperative therapy with a stable family environment having full support of all family members.

5. Patients with syndromic obesity, mental retardation and psychological disorder must be evaluated on individual merit.

The protocol also recommended to follow commonsense practical and step-wise intensification in the management option. The bariatric procedures should be done in regionalized specialized centers able to provide comprehensive preoperative and postoperative investigations with multidisciplinary team capable of providing long-term follow-up including the unique behavioral challenges of children and adolescents.

\section{Suggested surgical procedure for adolescents:}

Table-II outlines the recommended procedure(s) based on BMI and age of the patients. ${ }^{32-33}$

\section{Table-II}

\begin{tabular}{|c|c|c|c|}
\hline \multicolumn{4}{|c|}{ Surgical approach in obese adolescents. ${ }^{32-33}$} \\
\hline Group & Age (years) & $\operatorname{BMI}\left(\mathrm{kg} / \mathrm{m}^{2}\right)$ & Procedure(s) \\
\hline I & $13-19$ & $\begin{array}{l}\text { Less than or } \\
\text { equal to } 50\end{array}$ & LAGB or SG \\
\hline II & $13-15$ & Greater than 50 & SG \\
\hline III & $16-19$ & Greater than 50 & SG or RYGB \\
\hline
\end{tabular}

Patients aged 13 to 19 years with BMI less than or equal to $50 \mathrm{~kg} / \mathrm{m}^{2}$ are considered as group-I. LAGB or SG can be a good first choice for this group of patients because they have less obesity-related comorbidities. They respond well to the gradual increasing pattern of weight loss that occurs in LAGB.
Group-II comprises patients aged 13 to 15 years with BMI greater than $50 \mathrm{~kg} / \mathrm{m}^{2}$. This cohort of patients encounters problems with LAGB. They lose similar weight as their lower-BMI counterparts but the large amount of excess weight that remains and the slower rate of weight loss leads to frustration and unmet expectations. Further tightening of the gastric band to help accelerate weight loss often results in complications. A more aggressive procedure is therefore recommended, such as the SG, which would provide additional appetite suppression without the risk of micronutrient deficiencies along with an earlier and faster weight loss response.

Patients aged 16 to 19 years with BMI greater than 50 $\mathrm{kg} / \mathrm{m}^{2}$ are included in group-III. LAGB has limited effectiveness in this group for the same reason. It is likely that SG or RYGB would provide an earlier and faster weight loss. RYGB is technically more challenging compared to SG with a shallow learning curve. Also, the lower risk of micronutrient deficiencies associated with SG makes it an attractive option for this group. A failed $\mathrm{SG}$ is probably best salvaged by an RYGB.

\section{Suggested surgical procedure for children:}

In order to recommend a bariatric surgical procedure for obese children younger than 13 years, one must appreciate various age-specific physiologic and psychological issues. For instance, children younger than 13 years old have not completed their linear growth. Their BMI can improve when weight gain is arrested while the linear growth remains. Though it is not clear whether this process reduces the body fat mass and resolves obesity related comorbidities. The behavior of children under 13 years old is more malleable and shows better compliance. The non-surgical weight management program must be rigorously carried out under direct supervision of a professional multidisciplinary team. There are only few examples of bariatric procedures performed in obese children younger than 13 years old. At present, it is apparent that younger adolescents are more accepting the LAGB procedure. It has an excellent safety profile with reversibility. Its adjustability allows controlled caloric ingestion and maintains nutrition without malabsorption. Control of food ingestion in this group may allow them to undergo the longitudinal growth that leads to a normalization of their BMI without malnutrition. The adjustable gastric band can then be 
emptied, and if weight remains within an acceptable range, it could be easily removed.

\section{Bangladesh perspective:}

The rate of obesity in urban children and adolescents is increasing in Bangladesh and other developing countries though not as alarmingly as in developed world. In Bangladesh, the number of obese and overweight children has doubled from 1980 to 2013 while it has tripled in the USA during the same span of time. Nevertheless, the consequences and magnitude of the problem must not be underestimated because obesity, declared by World Health Organisation (WHO) as a disease, may be a cause of manifold comorbidities as already described. The treatment of obesity is likely to be expensive and we must focus on its prevention. Exclusive breast feeding (EBF) from birth to 6 months of age is an important way to help prevent infants from becoming overweight and obese. Strict antenatal checkup of pregnant mothers and prompt and effective treatment of gestational diabetes may also help prevent obesity in the newborn. Besides, healthy complimentary foods and a well-planned family diet play a paramount role in preventing obesity. Avoidance of sedentary lifestyle, eating junk and fast-food and engagement in increased physical activities are also pivotal for prevention of obesity. Much concerted efforts are required to put a rein on the alarming rise of obesity. The World Health Assembly in 2014 adopted the "Global Action Plan"; we must also develop a political commitment to advance the implementation of the WHO Global Strategy.

The pediatric bariatric surgery is still at the very early stage even in the developed world. In Bangladesh, like other developing countries, bariatric surgery in children and adolescents will require more time and further evaluation to set on. We need to establish a dedicated centre for weight-loss surgery -initially for adults and then it may be scalable to the needs of the children and adolescents.

\section{Conclusion:}

Bariatric surgical decision for pediatric obesity is never easy. One must not lose sight of the fact that treatment paradigm should be developmentally appropriate, and perhaps step-wise, beginning with conservative, safer and potentially reversible options. The risk-benefit ratio of weight loss surgery must be meticulously assessed before assigning one procedure to an obese patient so that benefit outweighs the risks. The development of stratified protocols of increasing intensity should be individualized for each patient based on their disease severity and risk factors. The goal is not only to achieve dramatic and sustained weight loss and to resolve the associated comorbidities but also to use a bariatric procedure which is most effective with negligible and minimum long-term outcome considering the growth and maturation potential of the children and adolescents. Success of pediatric bariatric surgery lies in the event of normal physiologic progression of a child through adolescence and adulthood that can only be ensured by selecting a bariatric procedure grounded upon sound physiological concept. Absence of restrictive food ingestion and malabsorption, and allowing normal life and growth without the necessity of life-long medications, nutritional support and surveillance are essential elements of an ideal bariatric procedure for obese children and adolescents.

\section{References:}

1. Inge TH, Zeller MH, Lawson L, Daniels SR. Critical appraisal of the evidence supporting bariatric surgery for weight management in adolescence. J Pediatr 2005; 147: $10-19$

2. Inge TH, Xanthakos AS, Zeller MH. Bariatric surgery for pediatric extreme obesity: now or later? Int J Obes 2007; 31: $1-14$

3. Franz MH, Van Wormer JJ, Crain AL, et al: Weight loss outcomes: a systematic review and meta-analysis of weight-loss clinical trials with a minimum 1 year followup. J AM Diet Assoc 2007; 107: 1755-67.

4. Martin LF. The evolution of surgery for morbid obesity. In: Martin LF, editor. Obesity surgery. New York: McGrawHill; 2004. p.15-48.

5. Lakka HM, Bouchard C. Etiology of obesity. In: Buchwald H, Cowan G, Pories W, eds. Surgical management of obesity. Philadelphia: Saunders; 2007. p.17-26.

6. Ogden CL, Carroll MD, Curtin LR, et al. Prevalence of high body mass index in US children and adolescents, 2007-2008. JAMA 2010; 303(3): 242-49

7. Popkin B. Global dynamics in childhood obesity: reflections on a life of work in the field. In: Freemark M, editor. Pediatric obesity. N Y, Springer; 2010. p.3-12.

8. Freedman DS, Mei Z, Srinivasan SR, et al. Cardiovascular risk factors and excess adiposity among overweight children and adolescents: The Bogalusa Heart Study. J Pediatr 2007; 150: 12-17. 
9. Ng M, Fleming T, Robinson M, Thomson B et al. Global, regional, and national prevalence of overweight and obesity in children and adults during 1980-2013: a systematic analysis for the Global Burden of Disease Study 2013. Lancet 2014; 384: 766-81.

10. Bulbul T, Hoque M. Prevalence of childhood obesity and overweight in Bangladesh findings from a countrywide epidemiological study.BMC Pediatr, 2014; 14:86.

11. Oken E, Gillman MW. Fetal origins of obesity. Obes Res 2003; 11: 496-506.

12. Sorensen HT, Sabroe S, Rothman KI, et al. Relation between weight and length at birth and body mass index in young adulthood:Cohort study.BMJ 1997;315:1137.

13. Bavdekar A, Yajnik CS, Fall CH, et al. Insulin resistance syndrome in 8-year-old Indian children: Small at birth, big at 8 years, or both? Diabetes 1999;48:2422-29.

14. Whitaker RC, Wright JA, Pepe MS, et al. Predicting obesity in young adulthood from childhood and parental obesity. N Engl J Med 1997; 337: 869-73.

15. Pinhas-Hamiel O, Zeitler P. The global spread of type-2 diabetes mellitus in children and adolescents. J Pediatr 2005; 146: 693-700.

16. Igne TH, Miyano G, Bean J, et al. Reversal of type-2 diabetes mellitus and improvement in cardiovascular risk factors after surgical weight loss in adolescents. Pediatrics 2009; 123: 214-22.

17. Pratt JS, Lenders CM, Dionne EA, et al. Best practice updates for pediatric/adolescent weight loss surgery. Obes Silver Spring 2009; 17: 901-10.

18. Shah AS, Khoury PR, Dolan LM, et al. The effects of obesity and type-2 diabetes mellitus on cardiac structure and function in adolescents and young adults. Diabetologia 2011; 54(4): 722-30.

19. Chitturi S, Farrell GC, George J. Non-alcoholic steatohepatitis in the Asia-Pacific region: Future shock? J Gastroenterol Hepatol 2004; 19: 368-74.

20. Kalra M, Inge $T$, Garcia V, et al. Obstructive sleep apnea in extremely overweight adolescents undergoing bariatric surgery. Obes Res 2005; 13: 1175-79.

21. Zeller MH, Modi AC, Noll JG, et al. Psychosocial functioning improves following adolescents bariatric surgery. Obes Silver Spring 2009; 17: 985-96.

22. Lee JM, Lim S, Zoellner, et al. Don't children grow out of their obesity? Weight transitions in early childhood. Clin Pediatr (Phila) 2010; 49(5): 466-69.

23. Garcia VF, De Maria EJ. Adolescent bariatric surgery: treatment delayed, treatment denied, a crisis invited. Obes Surg 2006; 16: 1-4.

24. Silber T, Randolph J, Robbins S. Long-term morbidity and mortality in morbidly obese adolescents after jejunoileal bypass. J Pediatr 1986; 108:318-22.
25. Greenstein RJ, Rabner JG. Is adolescent gastric-restrictive anti-obesity surgery warranted? Obes Surg 1995; 5: 138-44.

26. O’Brien PE, Sawyer SM, Laurie C, et al. Laparoscopic adjustable gastric banding in severely obese adolescents: A randomized trial. JAMA 2010; 303:519-26.

27. Treadwell JR, Sun F, Schoelles K. Systematic review and meta-analysis of bariatric surgery for pediatric obesity. Ann Surg 2008; 248:763-76.

28. Inge TH, Jenkins TM, Zeller M, et al. Baseline BMI is a strong predictor of nadir BMI after adolescent gastric bypass. J Pediatr 2010; 156:103-8.

29. Franco JV, Ruiz PA, Palermo M, et al. A review of studies comparing three laparoscopic procedures in bariatric surgery: sleeve gastrectomy, Roux-en-Y gastric bypass and adjustable gastric banding. Obes Surg 2011; 21(9): 1458-68.

30. Michalsky M, Reichard K, Inge T, et al. ASMBS pediatric committee best practice guidelines. Surg Obes Relat Dis 2012; 8(1): 1-7.

31. Michalsky M, Kramer RE, Fullmer MA, et al. Developing criteria for pediatric/adolescent bariatric surgery programs. Pediatrics 2011;128(Supp1 2): 65-70.

32. Fullmer MA, Abrams SH, Hrovat K, et al. Nutritional strategy for the adolescent patient undergoing bariatric surgery: report of a Working Group of the Nutrition Committee for the North American Society of Pediatric Gastroenterology, Hepatology and Nutrition and National Association of Children's Hospital and Related Institutions. J Pediatr Gastroenterol Nutr 2012;54(1): 125-35.

33. Barnett SJ. Contemporary surgical management of the obese adolescents. Curr Opin Pediatr 2011;23(3): 351-55.

34. Cunneen SA. Review of meta-analytic comparisons of bariatric surgery with a focus on laparoscopic adjustable gastric banding. Surg Obes Relat Dis 2008; 4(Suppl 3): 47-55.

35. Holterman AX, Browne A, Tussing L, et al. A prospective trial for laparoscopic adjustable gastric banding in morbidly obese adolescents: an interim report of weight loss, metabolic and quality of life outcomes. J Pediatr Surg 2010;45:74-79.

36. Xanthakos SA. Bariatric surgery for extreme adolescent obesity: indications, outcomes, and physiologic effects on the gut-brain axis. Pathophysiology 2008; 15(2): 135-46.

37. Lawson ML, Kirk S, Mitchell T, et al. One-year outcomes of Roux-en-Y gastric bypass for morbidly obese adolescents: a multicenter study from the Pediatric Bariatric Study Group. J Pediatr Surg 2006; 41:137-43. 
38. Clinical Issues Committee: Sleeve gastrectomy as a bariatric procedure. Surg Obes Relat Dis 2007; 3:573-76.

39. American Society for Metabolic and Bariatric Surgery. Updated position statement on sleeve gastrectomy as a bariatric procedure. Surg Obes Relat Dis 2010; 6(1): 1-5.

40. Nadler EP, Barefoot LC, Qureshi FG. Early results after laparoscopic sleeve gastrectomy in adolescents with morbid obesity. Oral presentation at the $7^{\text {th }}$ Annual Academic Surgical Congress, Las Vegas (NV), February 2012 .

41. Stimac D, Majanovic SK, Turk T, et al. Intragastric balloon treatment for obesity: results of a large single center prospective study. Obes Surg 2011; 21(5): 551-55.

42. Gersin KS, Torhstein RI, Tosenthal RJ, et al. Open-label, sham-controlled trial of an endoscopic duodenojejunal bypass liner for preoperative weight loss in bariatric surgery candidates. Gastrointest Endosc 2010; 71(6): 976-82.

43. Camilleri M, Toouli J, Herrera MF, et al. Selection of electrical algorithms to treat obesity with intermittent vagal block using an implantable medical device. Surg Obes Relat Dis 2009; 5(2): 224-29.

44. Shikora SA, Bergenstal R, Bessler M, et al. Implantable gastric stimulation for the treatment of clinically severe obesity: results of the SHAPE trial. Surg Obes Relat Dis 2009; 5(1): 31-37.

45. Brethauer SA, Harris JL, Kroh M, et al. Laparoscopic gastric placation for treatment of severe obesity. Surg Obes Relat Dis 2011;7(1): 15-22. 